\title{
Théologiques
}

\section{La création dans tous ses états}

\section{Walter Moser}

Volume 2, numéro 1, mars 1994

Création et créativité

URI : https://id.erudit.org/iderudit/602395ar

DOI : https://doi.org/10.7202/602395ar

Aller au sommaire du numéro

Éditeur(s)

Faculté de théologie de l'Université de Montréal

ISSN

1188-7109 (imprimé)

1492-1413 (numérique)

Découvrir la revue

Citer cet article

Moser, W. (1994). La création dans tous ses états. Théologiques, 2(1), 5-24.

https://doi.org/10.7202/602395ar d'utilisation que vous pouvez consulter en ligne.

https://apropos.erudit.org/fr/usagers/politique-dutilisation/ 
LIMINAIRE

\section{La création dans tous ses états}

Walter MOSER

Département de littérature comparée

Université de Montréal

En 1985 René Passeron affirme que «après une longue éclipse, le mot création réapparaît " ${ }^{1}$. Ce constat fait écho à celui de Mikel Dufrenne, formulé en 1981: «Dans l'esthétique la plus récente, le mot création a cessé d'être suspect » ${ }^{2}$. Un tabou a été levé, un mot se trouve réinséré dans le débat, un concept fait retour et s'offre à nouveau comme le point focal d'une discussion. Ce débat n'a en réalité jamais cessé, mais il a suivi d'autres voies de conceptualisation et a emprunté d'autres couvertures lexicales.

Le dossier présenté ici sur la question de la création est redevable de cette nouvelle dynamique. Il bénéficie de cette liberté nouvellement acquise d'un mot en occupant l'espace interdisciplinaire ainsi réouvert. En fait, il s'agit plutôt de réactiver un débat, que de l'instaurer. Passeron parle bien d'une éclipse, c'est-à-dire d'une disparition temporaire, que le mot "création" aurait subie. Et Dufrenne indique une piste pour expliquer cette éclipse: «les connotations théologiques du mot (...) déconseillaient son emploi " 3 . On voit bien que ce constat n'a de validité que sectorielle, car le lieu d'où parlent Passeron et Dufrenne est celui de l'esthétique. La portée de leur regard est ainsi limitée à un champ spécifique et ne leur

1 «Pour une approche 'poïétique' de la création», dans Encyclopaedia Universalis. Symposium, Les Enjeux, 1985, p. 149.

2 «Aujourd'hui encore, la création» dans Mikel DUFRENNE, Esthétique et philosophie. Tome 3, Paris, Klincksieck, 1981, p. 67.

3 Ibid. 
permet pas d'articuler l'interaction qu'entretient le terme qui nous intéresse avec d'autres champs ${ }^{4}$.

Nous soutenons ici que leur constat d'une disparition temporaire, tout intéressant et pertinent qu'il soit en esthétique, n'est cependant valable que pour ce regard limité. La question de la création n'a en réalité jamais cessé de se poser, la circulation du mot création peut être retracée sans interruption. À condition qu'on ouvre le champ de vision et qu'on ne se cantonne pas dans un secteur particulier, ou dans un discours spécialisé ou encore dans un champ pratique spécifique.

Dans ce numéro thématique, c'est ce travail d'ouverture qui sera entrepris avec le mot et le concept de création. Il est vrai que les cinq textes présentés ici ne sauraient couvrir la totalité du champ et représentent à leur tour un choix limité. Ils ouvrent néanmoins un vaste éventail qui va de l'exégèse du récit de la création biblique (Robert David) jusqu'à sa contrepartie scientifique de l'astrophysique moderne ${ }^{5}$ (Gilles Beaudet), en passant par la théologie (Pierre Gisel), le mouvement du créationnisme américain (Harvey Sarles) et par la conceptualisation de la créativité humaine (Hans Ulrich Gumbrecht). Il s'agit de baliser un lieu de réflexion et de proposer un débat qui relie entre eux ces différents espaces de mise en discours de la création. L'objectif étant d'une part de faire apparaître leurs interactions, leurs liens souterrains quand les passerelles explicites sont coupées ou interdites, d'autre part d'explorer leur profondeur historique et les parcours de leur développement se traçant souvent en chassé-croisé.

La démarche des articles présentés ici est, elle aussi, plurielle. Elle relève tout d'abord de la philologie dans la mesure où ce qui est travaillé c'est l'histoire d'un mot ou d'une famille de mots: créer, création, créativité. Il sera utile, au niveau lexématique, de commencer par préciser la signification de ces trois mots, ne fût-ce que de manière schématique:

créer: - au sens absolu: faire advenir ex nihilo, instaurer;

- au sens relatif: façonner, donner forme à un matériau prédonné;

4 Plus exactement l'approche poïtique de la création proposée par Passeron fait des incursions dans plusieurs disciplines, mais s'arrête abruptement devant la théologie: "Passe encore en théologie théorique - dont je n'ai pas à parler ici...» (p. 154).

5 Il s'agit en fait plutôt de la dénégation d'un récit de la création, parce que la science moderne doit se priver de l'avantage qu'a le mythe et qui consiste dans la traduction narrative de ce qui a toujours déjà eu lieu en un événement unique et fondateur. 
création: - l'acte de créer;

- la chose créée, la créature;

créativité: - la faculté de créer attribuée à un sujet appelé à assumer le rôle de créateur.

La démarche relève ensuite également et nécessairement de l'analyse et de l'histoire des discours, puisqu'il ne saurait y avoir de mots hors contexte et que les mots ne nous sont accessibles que dans des usages particuliers et dans des situations d'énonciation concrètes, c'est-à-dire toujours déjà mis en discours.

Une autre dimension du travail proposé renvoie à l'histoire des idées, ou à sa variante allemande la Begriffsgeschichte - qu'on traduirait littéralement par histoire des concepts ${ }^{6}$. On s'intéressera en fait également au processus de conceptualisation - surtout Gumbrecht qui traite ici des efforts récents pour donner à "créativité" la respectabilité d'un concept psychologique, pédagogique, etc. Le premier geste en histoire des concepts consiste à reconnaître que mot et concept ne coïncident pas et que, par conséquent, l'histoire du concept et l'histoire du mot sont souvent dissociées. Le chercheur doit donc considérer diverses couvertures lexicales pour retracer l'évolution d'un même concept. Inversement, les parcours de l'histoire d'un mot peuvent traverser divers champs conceptuels.

En outre, l'exégèse et la démarche herméneutique sous-tendront toutes les contributions réunies ici, même si elles ne s'engagent pas explicitement dans un travail d'ordre interprétatif. C'est que la tradition judéochrétienne - sécularisée elle deviendra "tradition occidentale» - se situe en aval des textes culturellement fondateurs de la Bible et que, dans tous les débats sur la création, résonnent encore les échos plus ou moins lointains de l'interprétation de ces textes. J'essaierai de montrer que même là où ce lien est volontairement rompu, il n'en est pas moins efficace - ne fût-ce que par dénégation.

Finalement, les approches évoquées ici, qui relèvent toutes des "sciences du langage" comportent des dimensions qui renvoient tantôt à des contextes institutionnels et politiques (c'est explicitement le cas dans le créationnisme scientifique présenté ici par Sarles), tantôt, dans la pédagogie démocratisante qui, selon Gumbrecht, a pris en charge le concept de créativité, à des traditions culturelles (la raison instrumentale

6 Dont le représentant paradigmatique, aujourd'hui, est Hans Blumenberg. 
moderne qui, selon Gisel et David, s'autorise d'une lecture du récit biblique de la création).

Afin de faire apparaître l'appartenance des études réunies ici à une même problématique, commençons par esquisser la situation actuelle de la question de la création. En exégèse biblique, les récits de la création? n'ont cessé de susciter des relectures et alimentent un travail d'interprétation continu et intarissable. Comme David le montre dans sa contribution, ce travail exégétique s'est intensifié dans les dernières dix années; et ceci en fonction de nouveaux enjeux et intérêts que les interprètes apportent au texte à partir de leur propre situation historique, sociale et culturelle. Loin de rencontrer de l'indifférence et même de tomber en désuétude, le texte premier fait l'objet de réinterprétations fortement appropriantes, voire critiques qui voient les interprètes prendre une part de plus en plus active dans la constitution du sens. L'exégèse donne ainsi lieu à, et va de pair avec, une espèce de critique idéologique et de réajustement qui se fait à l'intérieur de la théologie de la création, telle qu'on la trouve exposée dans le texte signé par Gisel. Le procès théologique de l'anthropocentrisme rejoint ainsi la critique de la modernité, et plus spécifiquement celle de la raison instrumentale menée par les penseurs de l'École de Francfort, et débouche, entre autres choses, sur la quête d'une nouvelle éthique à l'égard du créé.

Sarles montre que, à l'opposé de cette réinterprétation critique du texte de la création, se développe simultanément un retour au texte biblique. La plus grande fidélité littérale est postulée et pratiquée, le texte redevient une source d'autorité absolue, au point de pouvoir concurrencer le savoir scientifique produit dans un autre lieu discursif et institutionnel. La séparation entre croire et savoir, telle qu'opérée dans la modernité occidentale, se trouve ainsi révoquée. Le texte biblique est proposé comme véhiculant une vérité qui ne cède rien à la science, ce qui permet aux "créationnistes" de faire du récit de la création biblique un rival sérieux des discours scientifiques, et de contester le bannissement de la Bible comme autorité non seulement morale mais aussi scientifique par l'école publique instaurée par l'État.

7 En tout premier lieu il s'agit de Genèse 1-3, mais la question de la création ne se limite pas à ce choix textuel tout aussi restreint que célèbre. 


\section{Détour historique}

C'est comme si se rejouait aujourd'hui, à signes inversés, le conflit entre théologie et sciences qui marquait l'émancipation de l'activité scientifique par rapport à sa mise en tutelle par les instances ecclésiastiques. Cetre évolution devait, vers la fin du XVIII e siècle mener à l'autonomie des sciences et, à partir du XIX ${ }^{e}$ siècle, augmenter leur autorité et prestige dans les sociétés occidentales. Buffon a joué un rôleclé dans ce processus, et il sera intéressant de rappeler ici quelques aspects de sa lutte en faveur d'une activité scientifique autonome afin de montrer l'impact qu'a eu cette lutte sur les mises en discours ultérieures de la notion de création. Ceci nous permettra également de re-connaître enjeux et arguments dans les débats actuels qui ont lieu autour du créationnisme et d'apprécier l'effet d'inquiétante familiarité qui s'en dégage.

Suite à la publication de sa Théorie de la Terre en 1749, Buffon fut interpellé par la Faculté de théologie de la Sorbonne qui lui enjoignit de s'expliquer sur les divergences entre son récit savant de l'histoire de la planète Terre et le récit biblique de la création. La réponse de Buffon était conciliante et visait à reconnaître l'autorité et de la Bible et de ses exégètes attitrés tout en aménageant la possibilité de faire coexister les deux récits dans la même société. L'enjeu était important: il y allait de la séparation de croire et savoir, sans contester directement la vérité accordée au texte biblique ni l'autorité des exégètes qui géraient le sens du texte sacré sur les premières choses du monde.

Ainsi Buffon, quand il reprit le récit de la formation de la Terre dans les années ' 70 dans l'ouvrage qui, après une longue gestation, devait être publié sous le titre de Époques de la nature, fit-il preuve d'une grande prudence. C'est que ses recherches subséquentes à la Théorie de la Terre n'avaient fait que creuser l'écart entre les deux récits sur l'origine de la Terre. C'est tout particulièrement l'âge de la Terre, s'établissant selon une lecture littérale de la Bible à quelques milliers d'années, qui atteignait selon les observations scientifiques plusieurs centaines de milliers d'années ${ }^{8}$. Comment affirmer désormais la vérité scientifique sans contredire la vérité révélée dans le texte biblique et affronter, en conséquence, les autorités ecclésiastiques? Buffon opta encore une fois pour une stratégie de conciliation. Elle avait cependant ceci de subtil, ou

8 Un chiffre bien modeste à la lumière des 15 milliards d'années que les astrophysiciens accordent aujourd'hui à l'univers. 
de provoquant, que, pour opérer cette conciliation, il s'installa dans ce qui pouvait être considéré à l'époque comme la chasse gardée de l'Église: l'exégèse biblique.

En fait, dans le Discours premier qui figure comme une introduction à Époques de la nature, Buffon réinterpréta le récit biblique de la création, plus exactement Genèse 1 et 2 . Il ne s'agit pas d'exposer ici tout le travail qu'il fait en tant qu'exégète, mais plutôt de montrer comment sa stratégie interprétative lui permet de légitimer sa démarche scientifique du point de vue théologique, c'est-à-dire d'instaurer un récit concurrentiel à celui de la Bible tout en l'appuyant sur l'autorité du texte sacré.

Buffon commence par exposer toutes les évidences empiriques qui l'autorisent à distinguer six époques dans la formation de la Terre et à accorder à chacune une durée respectable. Mais voilà qu'une objection sérieuse se lève, ou plutôt qu'il prévient une objection que la Faculté de théologie pourrait lui faire

Mais avant d'aller plus loin, hâtons-nous de prévenir une objection grave, qui pourrait même dégénérer en imputation. Comment accordezvous, dira-t-on, cette haute ancienneté que vous donnez à la matière, avec les traditions sacrées, qui ne donnent au monde que six ou huit mille ans ? ${ }^{9}$

C'est cette question rhétorique qui appellera le travail exégétique proprement dit. Buffon le concentrera sur deux éléments du texte biblique - le verbe «créer» et le substantif «jour" - dont il tient à préciser le sens. Pour ce faire il mobilise tout un arsenal d'arguments herméneutiques qui relèvent tantôt de la philologie et de l'analyse contextuelle, tantôt du postulat qu'une logique rationnelle régit le texte à interpréter ${ }^{10}$. 11 met cet aspect du texte en avant en se servant d'une métaphore médicale ${ }^{11}$ :

9 BUFFON, Époques de la Nature. Edition critique établie par Jacques Roger. Paris, Éditions du Musée, 1962, p. 18.

10 J'ai analysé cette performance interprétative plus en détail dans «Buffon: exégète entre théologie et géologie», Strumenti Critici N.S. II/1, 1987, pp. 17-42.

11 Cetre métaphore de la «saine raison» est d'ailleurs reprise: «Ainsi pour entendre sainement ces premières paroles, il faut nécessairement suppléer un mot...» (p. 19). En elle résonne toute une tradition rationaliste de l'herméneutique postulant que n'a de sens que ce qui est bien formé selon les lois de la rationalité. Buffon donnera une dimension théologique à cette tradition en insistant que cette rationalité présidant à la production et compréhension des textes soit nécessairement commune aux instances divine et humaine. 
"Voyons donc; tâchons d'entendre sainement les premiers faits que l'Interprète divin nous a transmis au sujet de la création" (p. 18).

Quant à l'interprétation du verbe "créer", la stratégie de Buffon consiste à y creuser l'écart entre les deux significations distinguées plus haut. Dans son texte, la première est «tirer du néant» (p. 19), la seconde "produire des formes tirées de la matière créée précédemment" (p. 19). Il y aurait donc une signification sémantiquement forte qui correspond à la création ex nihilo et une signification sémantiquement plus faible qui comprend toute production de nouvelles formes à partir d'une matière ou d'un matériau donné. C'est cette distinction qui permet à Buffon d'intercaler un espace de temps entre la création initiale de la matière et celle, subséquente, des formes et surtout d'étaler cette dernière dans une durée très étendue. Car, si la première est un acte unique et absolu, pris en charge par le récit mythique qui traduit ce qui a toujours déjà été en un événement fondateur, la seconde peut être confiée au récit scientifique qu'est l' "histoire naturelle» du XVIII ${ }^{\mathrm{e}}$ siècle ${ }^{12}$.

Buffon n'admet aucun doute quant à la validité de son travail interprétatif:

Je vois donc clairement que non-seulement on peut, mais que même

l'on doit, pour se conformer au sens du texte de l'Écriture sainte, regarder la création de la matière en général comme plus ancienne que les productions particulières et successives de ses différentes formes (p. 19).

Et il va jusqu'à proposer une nouvelle traduction du texte sacré. En transformant la phrase "Au commencement Dieu créa le Ciel et la Terre" en "Au commencement Dieu créa la matière du ciel et de la terre", il précise le texte de la Bible dans le sens de son interprétation. Il distinguera désormais le geste premier, unique et fort de la création divine et le travail de la lente et progressive production de formes à partir de la matière déjà créée. Dans cetre deuxième phase de la création, dira-t-il, l'instance créatrice a "agi avec le temps" (p. 21) et "produit successivement" ses ouvrages (p. 21).

Le terrain est maintenant préparé pour une réinterprétation des six jours de la création divine:

12 Dans sa Théorie du ciel de 1755, Kant, alors partisan inconditionnel du mécanisme newtonien, éloigne également la main de Dieu de sa création en creusant l'écart entre la création de la matière par Dieu et le fonctionnement subséquent de l'Univers en vertu des lois mécaniques inscrites dans la matière. 
Que pouvons-nous entendre par les six jours que l'Écrivain sacré nous désigne si précisément en les comptant les uns après les autres, sinon six espaces de temps, six intervalles de durée? Et ces espaces de temps indiqués par le nom de jours, faute d'autres expressions, ne peuvent avoir aucun rapport avec nos jours actuels (...) Ce ne sont donc que six espaces de temps; l'Historien sacré ne détermine pas la durée de chacun, mais le sens de la narration semble la rendre assez longue, pour que nous puissions l'étendre autant que l'exigent les vérités physiques que nous avons à démontrer (p. 21).

C'est ainsi que Buffon mobilise l'autorité du texte biblique pour traduire les jours du récit biblique en des époques de l'histoire naturelle. Son incursion sur le terrain de ses adversaires réels et potentiels, l'exégèse biblique, lui aura permis de légitimer sa propre activité scientifique en la basant sur ce texte premier. Théologie et sciences se trouvent réconciliées en vertu d'une performance interprétative qui ne fait, en réalité, que jeter les bases de leur séparation définitive. Désormais elles pourront s'entendre à condition de respecter l'attribution de leurs champs spécialisés et fonctionnellement différenciés. Grâce à une exégèse qui s'éloigne de la littéralité du texte et qui en active le sensus spiritualis, c'est-à-dire le sens profond ${ }^{13}$, Buffon étend l'autorité de ce dernier jusqu'à ce que l'activité scientifique - en particulier le récit de la formation de la planète Terre - se trouve incluse dans le sens du texte. La ruse de Buffon consiste donc à favoriser le développement des sciences sous le bouclier protecteur du texte sacré, même s'il travaille en réalité à libérer la science de la tutelle de la théologie.

Buffon ne figure ici que pour illustrer des changements majeurs qui sont intervenus dans l'évolution de la notion de création, changements que, par ailleurs, il n'aurait pu effectuer à titre individuel. Sa figure exemplaire nous permet également de voir à l'oeuvre une logique d'argumentation qui nous aidera à comprendre ce qui arrive de nos jours dans le traitement de la question de la création, que ce soit dans la science officielle («l'évolutionnisme scientifique») ou dans le créationnisme scientifique.

13 Buffon plaide avec prudence pour cette liberté interprétative que voudrait annuler aujourd'hui la lecture littérale postulée par le créationnisme: «...ne jamais nous permettre de nous écarter de la lettre de cette sainte tradition que quand la lettre tue, c'est-à-dire, quand elle paraît directement opposée à la saine raison et à la vérité des faits de la Nature" (p. 22). 
En fait, pour le spécialiste du XVIII ${ }^{\mathrm{e}}$ siècle, le débat actuel du créationnisme tel que présenté ici par Sarles, produit un effet très net de déjà vu. C'est comme si le conflit de Buffon avec la Faculté de théologie se rejouait mutatis mutandis. Un certain argumentaire herméneutique est réactivé, la volonté de donner autorité au texte sacré sur ses concurrents profanes est réaffirmée. Mais tout cet effort est déployé pour faire marche arrière: il s'agit de révoquer la séparation entre croire et savoir, de rétablir la préséance du champ théologico-religieux sur le champ scientifique. On constate également le recours à une ruse argumentative: elle consiste à commencer par attribuer à "l'évolutionnisme scientifique" le statut d'une foi, à le démasquer ainsi comme un "croire" -- en lui déniant donc celui d'une vérité scientifique ("savoir») - pour ensuite le combattre sur ce terrain et dénoncer la mauvaise foi des institutions qui prennent la séparation entre État (responsable à travers le système d'éducation public de l'enseignement scientifique) et Église pour acquis.

L'attitude de la science contemporaine à l'égard de la création de l'univers - telle qu'elle est rapportée ici par Beaudet - relève également de la logique que nous venons de voir à l'œuvre chez Buffon. Il s'agit encore de séparer un acte de création premier, ponctuel et fort, des travaux de la nature dont la science peut fournir le récit. L'âge de l'univers, et par implication celui de la Terre qui était un enjeu majeur chez Buffon, a connu une augmentation «astronomique», ce qui a pour effet que le temps zéro de l'acte créateur premier est si éloigné dans le temps que le scientifique admet ne jamais pouvoir le connaître. La main de Dieu étant ainsi éloignée dans un temps mythique, le scientifique s'intéresse à l'Univers dès qu'il est abandonné aux lois naturelles dont la découverte est l'objectif premier des sciences naturelles. Mais contrairement à Buffon, le scientifique contemporain préfère renoncer entièrement au terme "création", de crainte de voir son travail contaminé par des connotations théologiques, même si la figure du big bang qui s'y substitue occupe la même position structurale et revêt la même fonction fondatrice d'événement initial et instaurateur.

\section{La création sécularisée}

Tant en sciences qu'en esthétique nous avons ainsi pu observer que le terme de création est devenu suspect à cause de ses connotations théologiques. Qu'il s'agisse d'expliquer la création de l'univers ou la création d'un monde artistique par un agent humain, l'enjeu est le même: comment continuer à parler de ce que "créer" désigne sans assumer les connotations de ses anciens usages - qui étaient mythiques, religieux, théologiques? Ou en d'autres termes: comment faire pour assurer que le 
discours théologique sur la création n'interfère pas avec celui des sciences et de l'esthétique? Nous avons déjà pu observer une réponse qui consiste à abandonner les mots "créer" et "création" et parler de ce qu'ils désignent sous d'autres couvertures lexicales. Cette réponse n'apporte cependant qu'une solution nominaliste à la question de savoir comment couper un mot de l'histoire de ses mises en discours.

En réalité ces questions et difficultés relèvent toutes d'une opération qui consiste à séculariser la création. Revenant au domaine de la création artistique, essayons de retracer la logique de cette opération. Un enjeu central de la sécularisation réside dans la transposition de l'acte de créer d'une instance divine à une instance humaine ou, comme le formule René Passeron «il s'agirait $(\ldots)$ de restituer le concept de création aux artistes, aux hommes " 14. La tâche consiste donc à conceptualiser l'acte créateur humain ainsi que le sujet auquel on attribue cet acte.

Séculariser veut dire faire passer une chose du sacré au profane, de l'état ecclésiastique à l'état laïque, du religieux au séculier. Il s'agit d'une transformation dont la réussite dépend de la possibilité de faire oublier dans son résultat l'état de départ. Ainsi, une théorie sécularisée de la création humaine aura tendance à attribuer la capacité de créer en propre au sujet humain et à supprimer le fait que, en réalité, la conceptualisation de cette capacité a résulté d'un transfert du divin à l'humain. La création humaine est en fair pensée selon un modèle divin, comme une copie ou imitation de la création divine.

Dans un geste qui sera d'inspiration généalogique, je propose de rétablir ici la mémoire de ce transfert frappé d'un oubli constitutif. Aussi formulerai-je l'hypothèse que, contrairement à ce que toute théorie sécularisée voudrait nous faire accroire, le concept de création humaine comporte toujours, comme deux moments inséparables, les termes que nous avons l'habitude de penser comme des oppositions irréconciliables: copier et créer, répéter et instaurer, imiter et faire advenir. Dans l'affirmation même de l'autonomie et de l'originalité de l'acte créateur humain, il y a la reprise des prédicats du créateur divin.

La création humaine étant en principe la réplique, la copie d'une création divine, on peut distinguer différentes manières de concevoir la création humaine, et ceci en suivant les distinctions introduites dans la manière de penser la création divine elle-même. Schématiquement donc, la création humaine apparaîtra comme l'imitation de

14 Op. cit., p. 154. 
1) l'acte créateur premier et fort: la création ex nihilo;

2) l'acte créateur second et faible: tirer de nouvelles formes d'une matière prédonnée. Dans le paradigme de l'imitation de la nature, le créateur humain imitera la natura naturans;

3) la créature qui est le produit de la création divine. Dans le paradigme de l'imitation de la nature, le créateur humain imitera la natura naturata .

Dans les deux premiers cas, la création artistique humaine adoptera les caractéristiques d'une poḯsis au sens le plus fort du terme ${ }^{15}$, tandis que, dans le troisième cas, elle relèvera davantage de la mimésis. Je me concentre ici sur les deux premiers cas où l'imitation humaine vise l'acte créateur, non pas le résultat de cet acte.

Dans le premier cas, on accordera au créateur humain le privilège de tirer un monde du néant et de se constituer ainsi comme l'origine d'un événement pur et absolu. Dans sa version païenne et pré-moderne, cette théorisation de la création artistique a recours aux figures de la possession, de l'inspiration et de la folie sacrée. Elle pose une instance divine s'installant dans un sujet humain qui sert de réceptacle et d'instrument au pouvoir créateur divin. L'instance humaine est alors vue comme possédée, habitée, inspirée d'une instance transcendante; elle est aliénée d'elle-même par une force supérieure agissant en elle, saisie d'une fureur ou d'une folie créatrice.

Dans sa version moderne, c'est-à-dire sécularisée, cette manière de penser la création artistique accordera à l'artiste humain le statut d'un créateur autonome. Il pourra non seulement faire comme Dieu, en faire autant que Dieu, mais rivalisera avec Dieu. La réappropriation de la figure mythique de Prométhée et de Pygmalion vers la fin du XVIII siècle s'inscrit entièrement dans cette conceptualisation orgueilleuse de la création humaine: le créateur humain lance un défi au créateur divin, que ce soit en lui volant le feu ou en insufflant la vie, il s'installe à sa place. Dans cette version, la théorie de la création artistique réinvestit le premier sens attribué par Buffon au verbe "créer" dans le récit biblique.

Dans le deuxième cas c'est le second sens explicité par Buffon qui se trouve réactivé. La création humaine est conçue comme l'imitation du potentiel créateur de la nature. On n'attribue plus au créateur humain le

15 Dans le sens d'un ressourcement étymologique du terme à partir du verbe grec "poïein»: faire, faire advenir. 
pouvoir de tirer l'être du néant, mais la capacité de créer de nouvelles formes et de nouveaux mondes à partir de matériaux prédonnés. Ce qui est imité est un acte créateur plus faible, second. La création apparaît ainsi comme un événement avec moins de force fondatrice, voire imperceptible. Transposée dans une figuration scientifique, elle relève moins du big bang que du clinamen. Ce dernier figure l'écart minimal par rapport à un état de choses donné, mais, écart premier et décisif, il est susceptible d'amener une transformation globale de cet état ${ }^{16}$. Dans cette théorisation le geste créateur est moins fort, bien que le potentiel créareur de ce geste soit presque illimité.

Les figurations modernes de ce type de création humaine sont celles de l'homo faber, de l'horloger au XVIII ${ }^{\mathbf{e}}$ siècle, de l'ingénieur et du constructeur au $\mathrm{XX}^{\mathrm{e}}$ siècle. Ce sont autant de figures pourvues d'un savoir faire technique très développé, souvent prises en charge par une éthique moderne de l'effort et du travail et animées par l'orgueil de la faisabilité humaine. Par elles l'acte créateur humain se trouve intrinsèquement branché sur une technique et une rationalité constructiviste au sens large du terme. Mais ce sont autant de figures qui appliquent leur pouvoir créateur à un matériau ou à un monde déjà existant. Par contre ce pouvoir ne s'efface plus derrière le résultat de sa mise en œuvre. Il s'exhibe dans son actualisation et se met lui-même en scène par une plus grande insistance sur le processus que sur le produit de la création.

Selon ce paradigme, créer est davantage transformer qu'instaurer. Plus précisément, ce qui est instauré n'est pas un nouvel univers matériel, mais une nouvelle configuration à partir de matériaux prédonnés. D'où la possibilité de prolonger historiquement la question de la création jusqu'aux procédés esthétiques modernistes du collage et du montage, et même jusqu'au procédé postmoderniste du recyclage, bien que la théorie moderne de la création y atteigne son moment aporétique ${ }^{17}$.

16 Clinamen - selon l'utilisation de ce terme en «science» par exemple par le Lucrèce du De Natura Rerum. Kant reprend cette figure dans sa Théorie du ciel de 1755 même s'il prend ses distances par rapport aux atomistes grecs et latins. C'est Harold Bloom qui, dans son Anxiety of Influence, a transposé ce terme de la science naturelle à la poétique.

17 Dans la mesure où, selon une certaine conception de la création, le recyclage apparaît comme son terme opposé. 


\section{La création démocratisée}

Les théories occidentales de la création, et plus particulièrement leurs versions modernes impliquent une théorie du sujet créateur. Elles sont même centrées sur la figure du créateur. Il s'agit de penser l'agent qui, en vertu de certaines qualités et facultés, est habilité à prendre en charge l'acte ou le processus créateur. Cet agent est la clé de la création dans la mesure où c'est en lui que réside le potentiel créateur, c'est de lui que part le travail créateur et c'est lui qui le mène à son terme.

Longtemps la mise en action de la création artistique était conçue comme partagée entre les instances divine et humaine sous la forme d'un agent humain possédé par une divinité qui exerçait son pouvoir créateur à travers lui. Conformément à ce qui vient d'être exposé, l'émergence de la modernité va de pair avec l'élaboration d'un sujet de la création artistique qui est caractérisé par son énergie créatrice propre, ce qui lui conferre une grande autonomie. Encore une fois, il est conçu à l'image du Créateur divin, mais le processus de sécularisation veut que son modèle sacré soit frappé d'oubli, afin qu'il puisse assumer à part entière, et en son propre droit, l'acte créateur. Du moins dans le domaine profane qu'est la création artistique.

Ce sont donc des qualités exceptionnelles, quasi-divines, qui font d'un sujet humain un créateur. Il est possible qu'il ait à subir des épreuves pour accéder à la création proprement dite, mais sa vocation, voire son élection, est basée sur des qualités supérieures au commun des mortels. Selon l'orientation spécifique de la théorisation et selon le mouvement artistique qui s'y manifeste, on le dira doué d'une force ou d'une énergie, d'une imagination, d'une richesse et d'une intensité de la vie intérieure, d'une intelligence, d'une capacité de figuration, d'une originalité, etc. hors du commun. L'important c'est que pour accéder au statut démiurgique du créateur humain, il faut être un sujet humain supérieur réunissant un ensemble de facultés et qualités extraordinaire. Le nom donné à ces individus exceptionnels est celui de génie.

L'histoire de la notion de génie (créateur) est suffisamment connue aujourd'hui pour qu'on n'ait pas à la retracer ici ${ }^{18}$. Qu'on le conçoive de manière positive comme le grand esprit et phare de l'humanité, ou négativement par la marginalisation sociale de l'artiste maudit, le génie

18 Qu'il suffise de renvoyer à l'aperçu synthétique qu'en donne Joachim SCHMIDT dans son ouvrage Die Geschichte des Geniegedankens (1750-1945). Darmstadt, Wissenschaftliche Buchgesellschaft Darmstadt, 1985. 
créateur désigne toujours un individu élu en vertu de ses qualités supérieures. Ainsi, selon une formulation proposée par Gumbrecht, le sujet créateur prend-il toujours appui sur un individu hyperbolique. Et il faut préciser: un individu masculin, ce qui est parfaitement en accord avec l'idée que le créateur humain est originairement conçu à l'image de Dieule-Père. L'Occident a en fait une longue tradition selon laquelle on attribue la force créatrice et le processus créateur à une instance masculine et qui postule, en conséquence, un sujet créateur masculin. Toute l'iconographie et toute la symbolisation de la création artistique sont imprégnées jusqu'à nos jours de cette sexuation de la création, qui est en soi un sujet d'analyse intéressant que je ne saurais cependant poursuivre ici plus loin.

Aux XVIII et XIXe siècles, le processus de création est l'affaire du génie, c'est-à-dire qu'il est le privilège d'un petit nombre de grands individus. Il est vrai qu'on entend ici et là une voix qui postule ou décrète que tout sujet humain est porteur d'une étincelle divine et participe de ce fait de la création divine. Mais le statut de sujet créateur n'est couramment accordé qu'aux artistes géniaux et reste donc la prérogative d'une espèce d'aristocratie artistique de naissance, puisque génie, on l'est et ne le devient pas.

Cette situation changea à partir de la deuxième moitié du XIX ${ }^{\mathrm{e}}$ siècle, sous l'influence de la démocratisation des institutions politiques et civiles dans les pays européens. Ce sont plus particulièrement les institutions pédagogiques, issues de ce processus historique, qui ont pris en charge l'idéal de la formation de l'individu comme le développement libre de son potentiel intellectuel et artistique. Il s'en dégagea une revendication anti-élitiste dans le domaine des arts. Elle se manifesta négativement comme l'abolition du privilège du perit nombre des génies créateurs. Le versant positif de la même tendance consiste en un transfert du discours des droits de l'homme du domaine politique à celui de la création artistique. Voici comment René Passeron formule ce transfert:

Le mouvement même de l'anti-art, dans la mesure où il a été une revendication anti-élitiste, impose la notion d'un droit collectif à la création, droit essentiel de toute personne dans l'affirmation de sa différence ${ }^{19}$.

Le cri de guerre sera désormais "nous sommes tous des génies" ou «il n'y a plus de génies». Le statut de sujet créateur doit devenir accessible à

19 Op. cit., p. 151. 
tout sujet humain, on doit pouvoir apprendre à devenir créateur. D'où l'intérêt récent pour explorer scientifiquement la possibilité de réveiller le sujet créateur qui somnole en tout individu humain. Cette approche scientifique de la création se manifeste tout particulièrement en psychologie et en pédagogie. Ces disciplines s'attachent en fait à l'exploration des qualités qui font de tout individu un sujet créateur.

L'ensemble de ces qualités est regroupé dans une faculté attribuée à tout être humain: la créativité. Les recherches sur la créativité -- dont Gumbrecht constate, non sans ironie, le développement pléthorique visent à définir la créativité, avant de proposer des méthodes pour la développer et l'appliquer à toutes sortes de champs pratiques.

On observe alors - Gumbrecht en fait la démonstration à travers les entrées de son fichier - une étrange relation entre une certaine indigence, sinon impossibilité conceptuelle du terme, et l'acharnement empirique pour en retracer les mécanismes et manifestations en vue d'améliorer les performances créatives et de faire bénéficier toute la population d'un apprentissage à la création 20 . Le message, implicite ou explicite, étant que, à partir des résultats d'une approche scientifique de la créativité, avec de la bonne volonté, et avec beaucoup de travail, un chacun peut accéder aux arcanes de la création, quel que soit son champ d'activités. Notons en passant que cette approche de la créativité fait ressortir la connotation "travail", "labeur» qui a accompagné de manière intermittente le terme "création" tout au long de son cheminement complexe retracé ici de manière très incomplète. L'idée que créer c'est travailler a alterné avec l'idée que la création humaine est une grâce accordée et œuvre comme par magie.

20 Je ne cite qu'un exemple: l'article qui a paru dans Le Devoir du 16 novembre 1993 et qui présentait, sous le titre "La science à l'aide de la créativité», un projet de recherche prestigieux qui se propose rien moins que d'uétudier l'essence de la créativité». Il veut pénétrer "le mystère de la création» en enregistrant et en analysant en détail la performance de créateurs-interprètes de haut niveau. L'objectif pratique étant le perfectionnement de la performance créative. Il n'est donc pas surprenant que, du pianiste, cette méthode puisse être transposée au «lanceur de javelot, qui est parvenu à améliorer grandement ses performances». Ce glissement, à l'intérieur même de la notion de création, de la musique vers le sport est l'indice d'un changement important qui devrait nous faire réfléchir autant que le romancier Robert Musil a fait réfléchir son personnage Ulrich, protagoniste du roman L'Homme sans qualités, sur sa découverte, dans les pages sportives de son quotidien, qu'on peut attribuer de la génialité à un cheval de course. 
Aujourd'hui la question de la création jouit en réalité d'une diffusion très large, bien au-delà des champs théologiques et artistiques. La vérification de ce fait est facile à faire si on sort des discours spécialisés et observe les pratiques discursives qui circulent sur la place publique que sont devenue nos médias écrits ou audiovisuels. La créativité est recherchée et débusquée partout, le champ d'action des créateurs actuels et futurs ne connaît plus de limites, puisqu'on les trouve dans la mode, en design, mais aussi en gestion, dans l'administration, en politique, sans oublier la cuisine, les sports, les activités que la langue française qualifie - par un terme intéressant dans ce contexte - de récréatives, etc. «La création d'emplois" est parmi les syntagmes les plus répandus qui circulent à l'heure actuelle dans les débats publics. Il renvoie d'ailleurs alternativement à de durs labeurs ${ }^{21}$ ou à un coup de génie qu'un intervenant inconnu aurait à exécuter comme par magie.

Par ailleurs, le mythe de la création semble aujourd'hui faire un retour déguisé en discours scientifique, évidemment sans que le mot "création" y soit associé. Il s'agit de l'intérêt que le grand public et les sciences humaines plus particulièrement portent depuis quelque temps à la théorie du chaos 22 . Élaborée de manière rigoureuse en mathématiques et en sciences, cette théorie, lorsqu'elle est transférée dans d'autres champs de savoir, connaît une réception très favorable qui va jusqu'à l'engouement. Cet accueil est marqué par une revalorisation positive de la notion de chaos qui apparaît alors comme comportant un potentiel créateur, comme la promesse de l'émergence de nouveaux univers. Même si ce nouveau développement ne donne pas lieu à ce qu'on pourrait formellement identifier comme des récits de création, il est hors de doute qu'il réactive ce qui, dans l'imaginaire culturel, occupe la place des mythes de la création. Il serait intéressant d'analyser les divers discours qui se greffent aujourd'hui sur la théorie du chaos pour y repérer un retour profane et séculaire de la mise en discours de la notion de création.

Nous trouvons donc, aujourd'hui, la création dans tous ses états. Sa démocratisation par une multiplication des sujets créateurs s'est doublée d'une banalisation. Non seulement tout le monde est potentiellement créateur, mais la création s'est répandue dans tous les champs d'activité.

21 À titre d'illustration cette phrase que Le Devoir du 20 mars 1994 met dans la bouche d'un politicien: «On travaille pour créer des emplois".

22 Parmi le grand nombre d'ouvrages récents portant sur cette question je ne mentionnerai que James GLEICK, Chaos: Making a New Science. New York, Viking, 1987 et Dahan DALMEDICO et al. (éds.), Chaos et déterminisme. Paris, Seuil, 1992. 
Discursivement parlant, ce phénomène se reconnaît à l'affaiblissement sémantique des mots et à leur usage dans des contextes qui relèvent du quotidien le plus banal.

Cette situation est à la fois intéressante et déroutante. Il est certain qu'à ce niveau de banalisation de la question, on se situe loin des modèles sacrés et de la figure forte du Créateur divin. Mais la création continue à être abordée à partir de l'agent créateur, qui serait à l'origine de l'acte créateur et responsable du niveau de sa performance créatrice. $\grave{A}$ travers tous les changements retracés ici, s'est donc maintenue une configuration du sujet dont on a déjà maintes fois annoncé la mort, ou dont on a au moins diagnostiqué la crise dans tous les domaines de la vie pratique. Comment se fait-il que cette configuration du sujet individuel autonome et fort survit dans le domaine de la création et bloque peut-être le chemin à de nouvelles amorces de conceptualisation?

Il y a pourtant eu des tentatives de sortir de cette tradition ou de la contourner. Je n'en mentionnerai que deux, rapidement.

En premier lieu il s'agit de la proposition marxiste, faite dans les années ' 60 et '70, de remplacer "création" par "production». Voici, à titre d'exemple, quelques lignes tirées de Pour une théorie de la production litteraire de Pierre Macherey ${ }^{23}$ :

Dire que l'écrivain, ou l'artiste est un créateur, c'est se placer dans la dépendance d'une idéologie humaniste.

Or l'art est une œuvre, non de l'homme, mais de ce qui la produit. [Les œuvres d'art] les hommes doivent les produire: non par la magie d'un avènement, mais par le moyen d'un travail réellement producteur. (...) l'artiste produit des oeuvres, dans des conditions déterminées: ouvrier non de lui-même, mais de cette chose qui lui échappe diversement et ne lui appartient jamais qu'après coup.

Les diverses «théories» de la création ont ceci de commun qu'elles traitent le problème de ce passage qu'est une fabrication en éliminant l'hypothèse d'une fabrication ou d'une production.

On comprend pourquoi, dans ces pages, le terme de création est supprimé, et remplacé systématiquement par celui de production (pp. 84-85).

23 Paris, François Maspéro, 1974 (ㄷ 1966). 
Ce déplacement de l'individu créateur vers le processus de production pensé comme un travail, une fabrication, a pour objectif la liquidation de l'idéologie humaniste et bourgeoise. En bannissant toute la famille des mots formés à partir du verbe "créer», on bannit la voie de cette idéologie qui continue à tésonner dans leur usage. Macherey propose ici plus qu'une opération nominaliste, puisqu'il a l'ambition de penser désormais le processus créateur dans un nouveau contexte conceptuel qui permettrait de penser différemment l'agencement de la création en faisant intervenir entre autres choses des conditionnements externes et des agents collectifs. Sans exclure la possibilité de concevoir, avec les nouvelles technologies intervenant de plus en plus massivement dans la création artistique, la possibilité d'un agencement mixte entre l'être humain et la machine.

Toutefois, cette nouvelle proposition n'est pas dépourvue de cécité. Sans l'expliciter elle réactive, par le biais des termes "travailler", «fabriquer», la version moderne de la réinterprétation sécularisante de l'Acte Créateur, y compris son éthique du travail et sa faisabilité utopique. Sans parler de la survivance dans une telle reconceptualisation de l'anthropocentrisme dominateur dont le procès est en cours, et pas seulement en théologie.

La seconde tentative nous ramène à René Passeron, et par là au début de cette présentation. Dans le domaine francophone, c'est en fait Passeron qui propose l'enquête la plus englobante du champ que nous venons de traverser. En 1985 il a résumé son entreprise déjà bien en cours dans «Pour une approche 'poíétique' de la création " 24.

L'entreprise de Passeron est ambitieuse dans la mesure où, en venant du champ esthétique, il souhaite inclure dans sa nouvelle science toute forme d'instauration opérée par des agents humains. Comme Macherey, il est soucieux de tenir en échec toute connotation théologique, mais contrairement à lui, il ne remplace pas pour autant le terme de création. Il le déplace plutôt vers celui de "poî́tique», ce qui lui permet de proposer un troisième lieu terminologique comme base d'opération conceptuelle: "La poiétique est l'étude scientifique et philosophique de cette instauration [des œuvres]» (p. 149).

Ayant emprunté le terme "poḯtique" à Paul Valéry comme «l'étude spécifique du faire» (p. 153), il effectue à la fois un transfert latéral (du

24 Il s'agit en fait d'une entreprise d'envergure, en marche depuis 1970 au moins. Elle a déjà produit de nombreux résultats qui sont largement diffusés par son propre organe de publication Recherches poïétiques. 
verbe «créer» au verbe «faire») et un transfert en profondeur en opérant un ressourcement étymologique lui permettant d'étendre la poïesis bien au-delà de la poésie ${ }^{25}$. Ce double geste l'autorise à accomplir deux choses: réunir sous un troisième terme l'opposition apparemment exclusive entre création et production et étendre l'esthétique sous ce nouvel intitulé «à tous les domaines extra-artistiques d'apparition des œuvres» (p. 153) 26 .

Ainsi, la poïétique n'aura pas seulement la prétention d'être une «interscience» ou une discipline transversale, mais aussi d'avoir une branche appliquée qui comporte peut-être la promesse de retombées plus-que-scientifiques.

Mais l'aspect décisif de cette approche réside dans la volonté de faire de la poḯtique une science et d'introduire dans un champ chargé de connotations de toutes sortes, un comportement scientifique. Je ne suis cependant pas convaincu que l'espèce de credo scientifique dont la présentation de Passeron est imprégnée réussira vraiment à neutraliser les charges idéologiques des termes «création" et "production» que nous avons vu émerger lors de notre parcours.

Chose certaine, dans sa réalisation textuelle le projet de Passeron absorbe et assume entièrement les deux moments importants qu'on vient de retracer ici: la sécularisation et la démocratisation. Vaste et globalisante, la nouvelle entreprise scientifique semble encore être animée, sinon englobée par ces deux enjeux majeurs et ne réussit guère à les objectiver de manière critique dans l'espoir d'arriver à repenser la création aujourd'hui.

Il faudra peut-être se tourner du côté de l'ouverture vers une conceptualisation différente que Gumbrecht indique plus qu'il ne l'accomplit dans son étude. L'enjeu principal consisterait alors en quelque sorte à sauter par-dessus l'ombre d'une forte tradition discursive qui a contracté l'habitude de ramener trop rapidement la création au sujet créateur et à la recherche de ses qualifications subjectives ou déterminations objectives. Dans cette forte tradition, génie démiurge et ouvrier travailleur, c'est-à-dire la version individualiste-bourgeoise et la

25 Cette réactivation de la racine grecque de «poésie» est à rapprocher de celle proposée par les biologistes Umberto Maturana et Francisco Varela qui parlent de la "auto-poiésis» des êtres vivants, terme qui a été repris dans la théorie des systèmes de dénomination luhmanienne, d'où il a fait son entrée dans les sciences humaines.

26 À titre d'exemple, il mentionne «les arts décoratifs, l'art-service, l'art-recherche, l'art commercial, l'art d'agrément» (p. 153). 
version marxiste se valent comme deux variantes du même paradigme. Gumbrecht propose de confier cette déprise par rapport à nos habitudes à une théorie du système. On pourrait débattre de cette décision que je considère être une question d'ordre stratégique. Ce qui me paraît décisif par contre c'est sa proposition de ne pas oublier l'événementialité radicale de l'Acte Créateur et, en conséquence, d'élaborer une conception de la création à partir des notions de contingence situationnelle et d'événement, plutôt qu'à partir des notions de sujet et d'action. La question ne serait plus de savoir à qui appartient l'acte créateur et en vertu de quelles qualités subjectives, mais: dans quelle situation la création peutelle survenir comme un événement radical? 